Article

\title{
Subordination Properties for Multivalent Functions Associated with a Generalized Fractional Differintegral Operator
}

\author{
Hanaa M. Zayed ${ }^{1, *(D)}$, Mohamed Kamal Aouf ${ }^{2}$ and Adela O. Mostafa ${ }^{2}$ \\ 1 Department of Mathematics, Faculty of Science, Menofia University, Shebin Elkom 32511, Egypt \\ 2 Department of Mathematics, Faculty of Science, Mansoura University, Mansoura 35516, Egypt; \\ mkaouf@mans.edu.eg (M.K.A.); adelaeg254@yahoo.com (A.O.M.) \\ * Correspondence: hanaa_zayed42@yahoo.com
}

Received: 19 January 2018; Accepted: 17 April 2018; Published: 24 April 2018

check for updates

\begin{abstract}
Using of the principle of subordination, we investigate some subordination and convolution properties for classes of multivalent functions under certain assumptions on the parameters involved, which are defined by a generalized fractional differintegral operator under certain assumptions on the parameters involved.
\end{abstract}

Keywords: differential subordination; $p$-valent functions; generalized fractional differintegral operator

JEL Classification: 30C45; 30C50

\section{Introduction and Definitions}

Denote by $\mathcal{A}(p)$ the class of analytic and $p$-valent functions of the form:

$$
f(z)=z^{p}+\sum_{n=1}^{\infty} a_{p+n} z^{p+n}(p \in \mathbb{N}=\{1,2, \ldots\} ; z \in \mathbb{U}=\{z \in \mathbb{C}:|z|<1\}) .
$$

For functions $f, g$ analytic in $\mathbb{U}, f$ is subordinate to $g$, written $f(z) \prec g(z)$ if there exists a function $w$, analytic in $\mathbb{U}$ with $w(0)=0$ and $|w(z)|<1$, such that $f(z)=g(w(z)), z \in \mathbb{U}$. If $g$ is univalent in $\mathbb{U}$, then (see $[1,2])$ :

$$
f(z) \prec g(z) \Leftrightarrow f(0)=g(0) \text { and } f(\mathbb{U}) \subset g(\mathbb{U}) .
$$

If $\varphi(z)$ is analytic in $\mathbb{U}$ and satisfies:

$$
H\left(\varphi(z), z \varphi^{\prime}(z)\right) \prec h(z),
$$

then $\varphi$ is a solution of (2). The univalent function $q$ is called dominant, if $\varphi(z) \prec q(z)$ for all $\varphi$. A dominant $\widetilde{q}$ is called the best dominant, if $\widetilde{q}(z) \prec q(z)$ for all dominants $q$.

Let ${ }_{2} F_{1}(a, b ; c ; z)(c \neq 0,-1,-2, \ldots)$ be the well-known (Gaussian) hypergeometric function defined by:

$$
{ }_{2} F_{1}(a, b ; c ; z):=\sum_{n=0}^{\infty} \frac{(a)_{n}(b)_{n}}{(c)_{n}(1)_{n}} z^{n}, z \in \mathbb{U},
$$

where:

$$
(\lambda)_{n}:=\frac{\Gamma(\lambda+n)}{\Gamma(\lambda)} .
$$

We will recall some definitions that will be used in our paper. 
Definition 1. For $f(z) \in \mathcal{A}(p)$, the fractional integral and fractional derivative operators of order $\lambda$ are defined by Owa [3] (see also [4]) as:

$$
\begin{gathered}
D_{z}^{-\lambda} f(z):=\frac{1}{\Gamma(\lambda)} \int_{0}^{z} \frac{f(\zeta)}{(z-\zeta)^{1-\lambda}} d \zeta \quad(\lambda>0), \\
D_{z}^{\lambda} f(z):=\frac{1}{\Gamma(1-\lambda)} \frac{d}{d z} \int_{0}^{z} \frac{f(\zeta)}{(z-\zeta)^{\lambda}} d \zeta \quad(0 \leq \lambda<1),
\end{gathered}
$$

where $f$ is an analytic function in a simply-connected region of the complex z-plane containing the origin, and the multiplicity of $(z-\zeta)^{\lambda-1}\left((z-\zeta)^{-\lambda}\right)$ is removed by requiring $\log (z-\zeta)$ to be real when $z-\zeta>0$.

Definition 2. For $f(z) \in \mathcal{A}(p)$ and in terms of ${ }_{2} F_{1}$, the generalized fractional integral and generalized fractional derivative operators defined by Srivastava et al. [5] (see also [6]) as:

$$
\begin{gathered}
I_{0, z}^{\lambda, \mu, \eta} f(z):=\frac{z^{-\lambda-\mu}}{\Gamma(\lambda)} \int_{0}^{z}(z-\zeta)^{\lambda-1} f(\zeta){ }_{2} F_{1}\left(\mu+\lambda,-\eta ; \lambda ; 1-\frac{\zeta}{z}\right) d \zeta(\lambda>0, \mu, \eta \in \mathbb{R}), \\
J_{0, z}^{\lambda, \mu, \eta} f(z):=\left\{\begin{array}{l}
\frac{d}{d z}\left\{\frac{z^{\lambda-\mu} \int_{0}^{z}(z-\zeta)^{-\lambda} f(\zeta)_{2} F_{1}\left(\mu-\lambda, 1-\eta ; 1-\lambda ; 1-\frac{\zeta}{z}\right) d \zeta}{\Gamma(1-\lambda)}\right\}(0 \leq \lambda<1), \\
\frac{d^{n}}{d z^{n}} J_{0, z}^{\lambda-n, \mu, \eta} f(z)(n \leq \lambda<n+1 ; n \in \mathbb{N}),
\end{array}\right.
\end{gathered}
$$

where $f(z)$ is an analytic function in a simply-connected region of the complex $z$-plane containing the origin with the order $f(z)=O\left(|z|^{\varepsilon}\right), z \rightarrow 0$ when $\varepsilon>\max \{0, \mu-\eta\}-1$, and the multiplicity of $(z-\zeta)^{\lambda-1}$ $\left((z-\zeta)^{-\lambda}\right)$ is removed by requiring $\log (z-\zeta)$ to be real when $z-\zeta>0$.

We note that:

$$
I_{0, z}^{\lambda,-\lambda, \eta} f(z)=D_{z}^{-\lambda} f(z)(\lambda>0) \text { and } J_{0, z}^{\lambda, \lambda, \eta} f(z)=D_{z}^{\lambda} f(z)(0 \leq \lambda<1),
$$

where $D_{z}^{-\lambda} f(z)$ and $D_{z}^{\lambda} f(z)$ are the fractional integral and fractional derivative operators studied by Owa [3].

Goyal and Prajapat [7] (see also [8]) defined the operator:

$$
S_{0, z}^{\lambda, \mu, \eta, p} f(z)=\left\{\begin{array}{l}
\frac{\Gamma(p+1-\mu) \Gamma(p+1-\lambda+\eta)}{\Gamma(p+1) \Gamma(p+1-\mu+\eta)} z^{\mu} J_{0, z}^{\lambda, \mu, \eta} f(z)(0 \leq \lambda<\eta+p+1 ; z \in \mathbb{U}), \\
\frac{\Gamma(p+1-\mu) \Gamma(p+1-\lambda+\eta)}{\Gamma(p+1) \Gamma(p+1-\mu+\eta)} z^{\mu} I_{0, z}^{-\lambda, \mu, \eta} f(z)(-\infty<\lambda<0 ; \quad z \in \mathbb{U}) .
\end{array}\right.
$$

For $f(z) \in \mathcal{A}(p)$, we have:

$$
\begin{aligned}
S_{0, z}^{\lambda, \mu, \eta, p} f(z) & =z_{3}^{p} F_{2}(1,1+p, 1+p+\eta-\mu ; 1+p-\mu, 1+p+\eta-\lambda ; z) * f(z) \\
& =z^{p}+\sum_{n=1}^{\infty} \frac{(p+1)_{n}(p+1-\mu+\eta)_{n}}{(p+1-\mu)_{n}(p+1-\lambda+\eta)_{n}} a_{p+n} z^{p+n} \\
(p & \in \mathbb{N} ; \mu, \eta \in \mathbb{R} ; \mu<p+1 ;-\infty<\lambda<\eta+p+1),
\end{aligned}
$$

where " $*$ " stands for convolution of two power series, and ${ }_{q} F_{s}\left(q \leq s+1 ; q, s \in \mathbb{N}_{0}=\mathbb{N} \cup\{0\}\right)$ is the well-known generalized hypergeometric function. 
Let:

$$
\begin{aligned}
G_{p, \eta, \mu}^{\lambda}(z) & =z^{p}+\sum_{n=1}^{\infty} \frac{(p+1)_{n}(p+1-\mu+\eta)_{n}}{(p+1-\mu)_{n}(p+1-\lambda+\eta)_{n}} z^{p+n} \\
(p & \in \mathbb{N} ; \mu, \eta \in \mathbb{R} ; \mu<p+1 ;-\infty<\lambda<\eta+p+1) .
\end{aligned}
$$

and:

$$
G_{p, \eta, \mu}^{\lambda}(z) *\left[G_{p, \eta, \mu}^{\lambda}(z)\right]^{-1}=\frac{z^{p}}{(1-z)^{\delta+p}}(\delta>-p ; z \in \mathbb{U}) .
$$

Tang et al. [9] (see also [10-15]) defined the operator $H_{p, \eta, \mu}^{\lambda, \delta}: \mathcal{A}(p) \rightarrow \mathcal{A}(p)$, where:

$$
\begin{aligned}
H_{p, \eta, \mu}^{\lambda, \delta} f(z) & =z^{p}+\sum_{n=1}^{\infty} \frac{(\delta+p)_{n}(p+1-\mu)_{n}(p+1-\lambda+\eta)_{n}}{(1)_{n}(p+1)_{n}(p+1-\mu+\eta)_{n}} a_{p+n} z^{p+n} \\
(p & \in \mathbb{N}, \delta>-p, \mu, \eta \in \mathbb{R}, \mu<p+1,-\infty<\lambda<\eta+p+1) .
\end{aligned}
$$

It is easy to verify that:

$$
z\left(H_{p, \eta, \mu}^{\lambda, \delta} f(z)\right)^{\prime}=(\delta+p) H_{p, \eta, \mu}^{\lambda, \delta+1} f(z)-\delta H_{p, \eta, \mu}^{\lambda, \delta} f(z),
$$

and:

$$
z\left(H_{p, \eta, \mu}^{\lambda+1, \delta} f(z)\right)^{\prime}=(p+\eta-\lambda) H_{p, \eta, \mu}^{\lambda, \delta} f(z)-(\eta-\lambda) H_{p, \eta, \mu}^{\lambda+1, \delta} f(z) .
$$

By using the operator $H_{p, \eta, \mu}^{\lambda, \delta}$, we introduce the following class.

Definition 3. For $A, B(-1 \leq B<A \leq 1), f \in \mathcal{A}(p)$ is in the class $\mathcal{T}_{p, \eta, \mu}^{\lambda, \delta}(A, B)$ if

$$
\frac{\left(H_{p, \eta, \mu}^{\lambda, \delta} f(z)\right)^{\prime}}{p z^{p-1}} \prec \frac{1+A z}{1+B z}(z \in \mathbb{U} ; p \in \mathbb{N}),
$$

which is equivalent to:

$$
\left|\frac{\frac{\left(H_{,, \eta, \mu}^{\lambda, \delta} f(z)\right)^{\prime}}{p z^{p-1}}-1}{B \frac{\left(H_{p, \eta}^{\lambda, \delta} f(z)\right)^{\prime}}{p z^{p-1}}-A}\right|<1(z \in \mathbb{U}) .
$$

For convenience, we write $\mathcal{T}_{p, \eta, \mu}^{\lambda, \delta}\left(1-\frac{2 \xi}{p},-1\right)=\mathcal{T}_{p, \eta, \mu}^{\lambda, \delta}(\xi) \quad(0 \leq \xi<p)$, which satisfies the inequality:

$$
\Re\left\{\frac{\left(H_{p, \eta, \mu}^{\lambda, \delta} f(z)\right)^{\prime}}{z^{p-1}}\right\}>\xi(0 \leq \xi<p) .
$$

In this paper, we investigate some subordination and convolution properties for classes of multivalent functions, which are defined by a generalized fractional differintegral operator. The theory of subordination received great attention, particularly in many subclasses of univalent and multivalent functions (see, for example, [13,15-17]).

\section{Preliminaries}

To prove our main results, we shall need the following lemmas.

Lemma 1. [18]. Let $h$ be an analytic and convex (univalent) function in $\mathbb{U}$ with $h(0)=1$. Additionally, let $\phi$ given by:

$$
\phi(z)=1+c_{n} z^{n}+c_{n+1} z^{n+1}+\ldots
$$


be analytic in $\mathbb{U}$. If:

$$
\phi(z)+\frac{z \phi^{\prime}(z)}{\sigma} \prec h(z)(\Re(\sigma) \geqslant 0 ; \sigma \neq 0),
$$

then:

$$
\phi(z) \prec \psi(z)=\frac{\sigma}{n} z^{-\frac{\sigma}{n}} \int_{0}^{z} t^{\frac{\sigma}{n}-1} h(t) d t \prec h(z),
$$

and $\psi$ is the best dominant of (6).

Denote by $P(\varsigma)$ the class of functions $\Phi$ given by:

$$
\Phi(z)=1+c_{1} z+c_{1} z^{2}+\ldots,
$$

which are analytic in $\mathbb{U}$ and satisfy the following inequality:

$$
\Re\{\Phi(z)\}>\varsigma(0 \leq \varsigma<1) .
$$

Using the well-known growth theorem for the Carathéodory functions (cf., e.g., [19]), we may easily deduce the following result:

Lemma 2. [19]. If $\Phi \in P(\varsigma)$. Then

$$
\Re\{\Phi(\varsigma)\} \geqslant 2 \varsigma-1+\frac{2(1-\varsigma)}{1+|z|}(0 \leq \varsigma<1) .
$$

Lemma 3. [20]. For $0 \leq \varsigma_{1}, \varsigma_{2}<1$,

$$
P\left(\varsigma_{1}\right) * P\left(\varsigma_{2}\right) \subset P\left(\varsigma_{3}\right)\left(\varsigma_{3}=1-2\left(1-\varsigma_{1}\right)\left(1-\varsigma_{2}\right)\right) .
$$

The result is the best possible.

Lemma 4. [21]. Let $\varphi$ be such that $\varphi(0)=1$ and $\varphi(z) \neq 0$ and $A, B \in \mathbb{C}$, with $A \neq B,|B| \leq 1, v \in \mathbb{C}^{*}$.

(i) If $\left|\frac{v(A-B)}{B}-1\right| \leq 1$ or $\left|\frac{v(A-B)}{B}+1\right| \leq 1, B \neq 0$ and $\varphi(z)$ satisfies:

$$
1+\frac{z \varphi^{\prime}(z)}{v \varphi(z)} \prec \frac{1+A z}{1+B z},
$$

then:

$$
\varphi(z) \prec(1+B z)^{v\left(\frac{A-B}{B}\right)}
$$

and this is the best dominant.

(ii) If $B=0$ and $|v A|<\pi$ and if $\varphi$ satisfies:

$$
1+\frac{z \varphi^{\prime}(z)}{v \varphi(z)} \prec 1+A z,
$$

then:

$$
\varphi(z) \prec e^{v A z},
$$

and this is the best dominant. 
Lemma 5. [2]. Let $\Omega \subset \mathbb{C}, b \in \mathbb{C}, \Re(b)>0$ and $\psi: \mathbb{C}^{2} \times \mathbb{U} \rightarrow \mathbb{C}$ satisfy $\psi(i x, y ; z) \notin \Omega$ for all $x, y \leq-\frac{|b-i x|^{2}}{2 \Re(b)}$ and all $z \in \mathbb{U}$. If $p(z)=1+p_{1} z+p_{2} z^{2}+\ldots$, is analytic in $\mathbb{U}$ and if:

$$
\psi\left(p(z), z p^{\prime}(z) ; z\right) \in \Omega,
$$

then $\Re\{p(z)\}>0$ in $\mathbb{U}$.

Lemma 6. [22]. Let $\psi(z)$ be analytic in $\mathbb{U}$ with $\psi(0)=1$ and $\psi(z) \neq 0$ for all $z$. If there exist two points $z_{1}, z_{2} \in \mathbb{U}$ such that:

$$
-\frac{\pi}{2} \rho_{1}=\arg \left\{\psi\left(z_{1}\right)\right\}<\arg \{\psi(z)\}<\frac{\pi}{2} \rho_{2}=\arg \left\{\psi\left(z_{2}\right)\right\},
$$

for some $\rho_{1}$ and $\rho_{2}\left(\rho_{1}, \rho_{2}>0\right)$ and for all $z\left(|z|<\left|z_{1}\right|=\left|z_{2}\right|\right)$, then:

$$
\frac{z_{1} \psi^{\prime}\left(z_{1}\right)}{\psi\left(z_{1}\right)}=-i\left(\frac{\rho_{1}+\rho_{2}}{2} \kappa\right) \text { and } \frac{z_{2} \psi^{\prime}\left(z_{2}\right)}{\psi\left(z_{2}\right)}=i\left(\frac{\rho_{1}+\rho_{2}}{2} \kappa\right)
$$

where:

$$
\kappa \geq \frac{1-|a|}{1+|a|} \text { and } a=i \tan \left(\frac{\rho_{2}-\rho_{1}}{\rho_{2}+\rho_{1}}\right) .
$$

\section{Properties Involving $\boldsymbol{H}_{p, \eta, \mu}^{\lambda, \delta}$}

Unless otherwise mentioned, we assume throughout this paper that $p \in \mathbb{N}, \delta>-p, \mu, \eta \in \mathbb{R}, \mu<$ $p+1,-\infty<\lambda<\eta+p+1,-1 \leq B<A \leq 1, \theta>0$, and the powers are considered principal ones.

Theorem 1. Let $f \in \mathcal{A}(p)$ satisfy:

$$
(1-\theta) \frac{\left(H_{p, \eta, \mu}^{\lambda, \delta} f(z)\right)^{\prime}}{p z^{p-1}}+\theta \frac{\left(H_{p, \eta, \mu}^{\lambda, \delta+1} f(z)\right)^{\prime}}{p z^{p-1}} \prec \frac{1+A z}{1+B z} .
$$

Then:

$$
\Re\left(\frac{\left(H_{p, \eta, \mu}^{\lambda, \delta} f(z)\right)^{\prime}}{p z^{p-1}}\right)^{\frac{1}{\tau}}>\left(\frac{\delta+p}{\theta} \int_{0}^{1} u^{\frac{\delta+p}{\theta}-1}\left(\frac{1-A u}{1-B u}\right) d u\right)^{\frac{1}{\tau}}, \tau \geq 1 .
$$

The estimate in (13) is sharp.

Proof. Let:

$$
\phi(z)=\frac{\left(H_{p, \eta, \mu}^{\lambda, \delta} f(z)\right)^{\prime}}{p z^{p-1}}(z \in \mathbb{U}) .
$$

Then, $\phi$ is analytic in $\mathbb{U}$. After some computations, we get:

$$
(1-\theta) \frac{\left(H_{p, \eta, \mu}^{\lambda, \delta} f(z)\right)^{\prime}}{p z^{p-1}}+\theta \frac{\left(H_{p, \eta, \mu}^{\lambda, \delta+1} f(z)\right)^{\prime}}{p z^{p-1}}=\phi(z)+\frac{\theta z \phi^{\prime}(z)}{\delta+p} \prec \frac{1+A z}{1+B z} .
$$

Now, by using Lemma 1, we deduce that:

$$
\frac{\left(H_{p, \eta, \mu}^{\lambda, \delta} f(z)\right)^{\prime}}{p z^{p-1}} \prec \frac{\delta+p}{\theta} z^{-\frac{\delta+p}{\theta}} \int_{0}^{z} t^{\frac{\delta+p}{\theta}-1}\left(\frac{1+A t}{1+B t}\right) d t
$$


or, equivalently,

$$
\frac{\left(H_{p, \eta, \mu}^{\lambda, \delta} f(z)\right)^{\prime}}{p z^{p-1}}=\frac{\delta+p}{\theta} \int_{0}^{1} u^{\frac{\delta+p}{\theta}}-1\left(\frac{1+A u w(z)}{1+B u w(z)}\right) d u,
$$

and so:

$$
\Re\left(\frac{\left(H_{p, \eta, u}^{\lambda, \delta} f(z)\right)^{\prime}}{p z^{p-1}}\right)>\left(\frac{\delta+p}{\theta} \int_{0}^{1} u^{\frac{\delta+p}{\theta}-1}\left(\frac{1-A u}{1-B u}\right) d u\right) .
$$

Since:

$$
\Re\left(\chi^{\frac{1}{\tau}}\right) \geq(\Re(\chi))^{\frac{1}{\tau}}(\chi \in \mathbb{C}, \Re\{\chi\} \geq 0, \tau \geq 1) .
$$

The inequality (13) now follows from (16) and (17). To prove that the result is sharp, let:

$$
\frac{\left(H_{p, \eta, \mu}^{\lambda, \delta} f(z)\right)^{\prime}}{p z^{p-1}}=\frac{\delta+p}{\theta} \int_{0}^{1} u^{\frac{\delta+p}{\theta}-1}\left(\frac{1+A u z}{1+B u z}\right) d u .
$$

Now, for $f(z)$ defined by (18), we have:

$$
(1-\theta) \frac{\left(H_{p, \eta, \mu}^{\lambda, \delta} f(z)\right)^{\prime}}{p z^{p-1}}+\theta \frac{\left(H_{p, \eta, \mu}^{\lambda, \delta+1} f(z)\right)^{\prime}}{p z^{p-1}}=\frac{1+A z}{1+B z}(z \in \mathbb{U}),
$$

Letting $z \rightarrow-1$, we obtain:

$$
\frac{\left(H_{p, \eta, \mu}^{\lambda, \delta} f(z)\right)^{\prime}}{p z^{p-1}} \rightarrow \frac{\delta+p}{\theta} \int_{0}^{1} u^{\frac{\delta+p}{\theta}-1}\left(\frac{1-A u}{1-B u}\right) d u,
$$

which ends our proof.

Putting $\theta=1$ and using Lemma 1 for Equation (15) in Theorem 1, we obtain the following example.

Example 1. Let the function $f(z) \in \mathcal{A}(p)$. Then, following containment property holds,

$$
\mathcal{T}_{p, \mu, \eta}^{\lambda, \delta+1}(A, B) \subset \mathcal{T}_{p, \mu, \eta}^{\lambda, \delta}(A, B) .
$$

Using (4) instead of (3) in Theorem 1, one can prove the following theorem.

Theorem 2. Let $f \in \mathcal{A}(p)$ satisfy

$$
(1-\theta) \frac{\left(H_{p, \eta, \mu}^{\lambda+1, \delta} f(z)\right)^{\prime}}{p z^{p-1}}+\theta \frac{\left(H_{p, \eta, \mu}^{\lambda, \delta} f(z)\right)^{\prime}}{p z^{p-1}} \prec \frac{1+A z}{1+B z} .
$$

Then:

$$
\Re\left(\frac{\left(H_{p, \eta, \mu}^{\lambda+1, \delta} f(z)\right)^{\prime}}{p z^{p-1}}\right)^{\frac{1}{\tau}}>\left(\frac{p+\eta-\lambda}{\theta} \int_{0}^{1} u^{\frac{p+\eta-\lambda}{\theta}-1}\left(\frac{1-A u}{1-B u}\right) d u\right)^{\frac{1}{\tau}}, \tau \geq 1 .
$$

The result is sharp.

Putting $\theta=1$ in Theorem 2, we obtain the following example. 
Example 2. Let the function $f(z) \in \mathcal{A}(p)$. Then, following inclusion property holds

$$
\mathcal{T}_{p, \mu, \eta}^{\lambda, \delta}(A, B) \subset \mathcal{T}_{p, \mu, \eta}^{\lambda+1, \delta}(A, B)
$$

For a function $f \in \mathcal{A}(p)$, the generalized Bernardi-Libera-Livingston integeral operator $F_{p, \gamma}$ is defined by (see [23]):

$$
\begin{aligned}
F_{p, \gamma} f(z) & =\frac{\gamma+p}{z^{p}} \int_{0}^{z} t^{\gamma-1} f(t) d t \\
& =\left(z^{p}+\sum_{k=1}^{\infty} \frac{\gamma+p}{\gamma+p+k} z^{p+k}\right) * f(z)(\gamma>-p) \\
& =z^{p}{ }_{3} F_{2}(1,1, \gamma+p ; 1, \gamma+p+1 ; z) * f(z)
\end{aligned}
$$

Lemma 7. If $f \in \mathcal{A}(p)$, prove that:

(i) $H_{p, \eta, \mu}^{\lambda, \delta}\left(F_{p, \gamma} f\right)=F_{p, \gamma}\left(H_{p, \eta, \mu}^{\lambda, \delta} f\right)$,

(ii)

$$
z\left(H_{p, \eta, \mu}^{\lambda, \delta} F_{p, \gamma} f(z)\right)^{\prime}=(p+\gamma) H_{p, \eta, \mu}^{\lambda, \delta} f(z)-\gamma H_{p, \eta, \mu}^{\lambda, \delta} F_{p, \gamma} f(z)
$$

Proof. Since

$$
\begin{aligned}
H_{p, \eta, \mu}^{\lambda, \delta}\left(F_{p, \gamma} f\right)= & {\left[z_{3}^{p} F_{2}(\delta+p, p+1-\mu, p+1-\lambda+\eta ; p+1, p+1-\mu+\eta ; z)\right] *\left(F_{p, \gamma} f\right) } \\
= & {\left[z^{p}{ }_{3} F_{2}(\delta+p, p+1-\mu, p+1-\lambda+\eta ; p+1, p+1-\mu+\eta ; z)\right] * } \\
& {\left[z^{p}{ }_{3} F_{2}(1,1, \gamma+p ; 1, \gamma+p+1 ; z) * f(z)\right] }
\end{aligned}
$$

and:

$$
\begin{aligned}
F_{p, \gamma}\left(H_{p, \eta, \mu}^{\lambda, \delta} f\right)= & z^{p}{ }_{3} F_{2}(1,1, \gamma+p ; 1, \gamma+p+1 ; z) *\left(H_{p, \eta, \mu}^{\lambda, \delta} f\right) \\
= & z^{p}{ }_{3} F_{2}(1,1, \gamma+p ; 1, \gamma+p+1 ; z) * \\
& {\left[z^{p}{ }_{3} F_{2}(\delta+p, p+1-\mu, p+1-\lambda+\eta ; p+1, p+1-\mu+\eta ; z) * f(z)\right] . }
\end{aligned}
$$

Now, the first part of this lemma follows. Furthermore,

$$
z\left(F_{p, \gamma} f(z)\right)^{\prime}=(p+\gamma) f(z)-\gamma F_{p, \gamma} f(z)
$$

If we replace $f(z)$ by $H_{p, \eta, \mu}^{\lambda, \delta} f(z)$ and using the first part of this lemma, we get $(21)$.

Theorem 3. Suppose that $p+\gamma>0, f \in \mathcal{T}_{p, \eta, \mu}^{\lambda, \delta}(A, B)$ and $F_{p, \gamma}$ defined by (20). Then:

$$
\Re\left(\frac{\left(H_{p, \eta, \mu}^{\lambda, \delta} F_{p, \gamma} f(z)\right)^{\prime}}{p z^{p-1}}\right)^{\frac{1}{\tau}}>\left((p+\gamma) \int_{0}^{1} u^{p+\gamma-1}\left(\frac{1-A u}{1-B u}\right) d u\right)^{\frac{1}{\tau}}, \tau \geq 1 .
$$

The result is sharp.

Proof. Let:

$$
\phi(z)=\frac{\left(H_{p, \eta, \mu}^{\lambda, \delta} F_{p, \gamma} f(z)\right)^{\prime}}{p z^{p-1}}(z \in \mathbb{U})
$$


Then, $\phi$ is analytic in $\mathbb{U}$. After some calculations, we have:

$$
\frac{\left(H_{p, \eta, \mu}^{\lambda, \delta} f(z)\right)^{\prime}}{p z^{p-1}}=\phi(z)+\frac{z \phi^{\prime}(z)}{p+\gamma} \prec \frac{1+A z}{1+B z} .
$$

Employing the same technique that was used in proving Theorem 1, the remaining part of the theorem can be proven.

Theorem 4. Let $-1 \leq B_{i}<A_{i} \leq 1(i=1,2)$. If each of the functions $f_{i} \in \mathcal{A}(p)$ satisfies:

$$
(1-\theta) \frac{H_{p, \eta, \mu}^{\lambda, \delta} f_{i}(z)}{z^{p}}+\theta \frac{H_{p, \eta, \mu}^{\lambda, \delta+1} f_{i}(z)}{z^{p}} \prec \frac{1+A_{i} z}{1+B_{i} z}(i=1,2),
$$

then:

$$
(1-\theta) \frac{H_{p, \eta, \mu}^{\lambda, \delta} F(z)}{z^{p}}+\theta \frac{H_{p, \eta, \mu}^{\lambda, \delta+1} F(z)}{z^{p}} \prec \frac{1+(1-2 \varrho) z}{1-z},
$$

where:

$$
F(z)=H_{p, \eta, \mu}^{\lambda, \delta}\left(f_{1} * f_{2}\right)(z)
$$

and:

$$
\varrho=1-\frac{4\left(A_{1}-B_{1}\right)\left(A_{2}-B_{2}\right)}{\left(1-B_{1}\right)\left(1-B_{2}\right)}\left[1-\frac{1}{2}{ }_{2} F_{1}\left(1,1 ; \frac{\delta+p}{\theta}+1 ; \frac{1}{2}\right)\right] .
$$

The result is possible when $B_{1}=B_{2}=-1$.

Proof. Suppose that $f_{i} \in \mathcal{A}(p)(i=1,2)$ satisfy the condition (25). Setting:

$$
p_{i}(z)=(1-\theta) \frac{H_{p, \eta, \mu}^{\lambda, \delta} f_{i}(z)}{z^{p}}+\theta \frac{H_{p, \eta, \mu}^{\lambda, \delta+1} f_{i}(z)}{z^{p}}(i=1,2),
$$

we have:

$$
p_{i}(z) \in P\left(\varsigma_{i}\right)\left(\varsigma_{i}=\frac{1-A_{i}}{1-B_{i}}, i=1,2\right) .
$$

Thus, by making use of the identity (3) in (29), we get:

$$
H_{p, \eta, \mu}^{\lambda, \delta} f_{i}(z)=\frac{\delta+p}{\theta} z^{p-\frac{\delta+p}{\theta}} \int_{0}^{z} t^{\frac{\delta+p}{\theta}-1} p_{i}(t) d t(i=1,2),
$$

which, in view of $F$ given by (27) and (30), yields:

$$
H_{p, \eta, \mu}^{\lambda, \delta} F(z)=\frac{\delta+p}{\theta} z^{p-\frac{\delta+p}{\theta}} \int_{0}^{z} t^{\frac{\delta+p}{\theta}-1} F(t) d t
$$

where:

$$
F(z)=(1-\theta) \frac{H_{p, \eta, \mu}^{\lambda, \delta} F(z)}{z^{p}}+\theta \frac{H_{p, \eta, \mu}^{\lambda, \delta+1} F(z)}{z^{p}}=\frac{\delta+p}{\theta} z^{-\frac{\delta+p}{\theta}} \int_{0}^{z} t^{\frac{\delta+p}{\theta}}-1\left(p_{1} * p_{2}\right)(t) d t .
$$

Since $p_{i}(z) \in P\left(s_{i}\right)(i=1,2)$, it follows from Lemma 3 that:

$$
\left(p_{1} * p_{2}\right)(z) \in P\left(\varsigma_{3}\right)\left(\varsigma_{3}=1-2\left(1-\varsigma_{1}\right)\left(1-\varsigma_{2}\right)\right) .
$$


Now, by using (33) in (32) and then appealing to Lemma 2, we have:

$$
\begin{aligned}
& \Re\{F(z)\}=\frac{\delta+p}{\theta} \int_{0}^{1} u^{\frac{\delta+p}{\theta}-1} \Re\left\{\left(p_{1} * p_{2}\right)(u z)\right\} d u \\
& \geqslant \frac{\delta+p}{\theta} \int_{0}^{1} u^{\frac{\delta+p}{\theta}-1}\left(2 \varsigma_{3}-1+\frac{2\left(1-\varsigma_{3}\right)}{1+u|z|}\right) d u \\
& >\frac{\delta+p}{\theta} \int_{0}^{1} u^{\frac{\delta+p}{\theta}-1}\left(2 \varsigma_{3}-1+\frac{2\left(1-\varsigma_{3}\right)}{1+u}\right) d u \\
& =1-\frac{4\left(A_{1}-B_{1}\right)\left(A_{2}-B_{2}\right)}{\left(1-B_{1}\right)\left(1-B_{2}\right)}\left[1-\frac{\delta+p}{\theta} \int_{0}^{1} u^{\frac{\delta+p}{\theta}-1}(1+u)^{-1} d u\right] \\
& =1-\frac{4\left(A_{1}-B_{1}\right)\left(A_{2}-B_{2}\right)}{\left(1-B_{1}\right)\left(1-B_{2}\right)}\left[1-\frac{1}{2}{ }_{2} F_{1}\left(1,1 ; \frac{\delta+p}{\theta}+1 ; \frac{1}{2}\right)\right]=\varrho \text {. }
\end{aligned}
$$

When $B_{1}=B_{2}=-1$, we consider the functions $f_{i}(z) \in \mathcal{A}(p)(i=1,2)$, which satisfy (25), are defined by:

$$
H_{p, \eta, \mu}^{\lambda, \delta} f_{i}(z)=\frac{\delta+p}{\theta} z^{p-\frac{\delta+p}{\theta}} \int_{0}^{z} t^{\frac{\delta+p}{\theta}-1}\left(\frac{1+A_{i} t}{1-t}\right) d t(i=1,2) .
$$

Thus, it follows from (32) that:

$$
\begin{aligned}
& F(z)=\frac{\delta+p}{\theta} \int_{0}^{1} u^{\frac{\delta+p}{\theta}-1}\left[1-\left(1+A_{1}\right)\left(1+A_{2}\right)+\frac{\left(1+A_{1}\right)\left(1+A_{2}\right)}{(1-u z)}\right] d u \\
= & 1-\left(1+A_{1}\right)\left(1+A_{2}\right)+\left(1+A_{1}\right)\left(1+A_{2}\right)(1-z)^{-1}{ }_{2} F_{1}\left(1,1 ; \frac{\delta+p}{\theta}+1 ; \frac{z}{z-1}\right) \\
\rightarrow & 1-\left(1+A_{1}\right)\left(1+A_{2}\right)+\frac{1}{2}\left(1+A_{1}\right)\left(1+A_{2}\right){ }_{2} F_{1}\left(1,1 ; \frac{\delta+p}{\theta}+1 ; \frac{1}{2}\right) \text { as } z \rightarrow-1,
\end{aligned}
$$

which evidently ends the proof.

Theorem 5. Let $v \in \mathbb{C}^{*}$, and let $A, B \in \mathbb{C}$ with $A \neq B$ and $|B| \leq 1$. Suppose that:

$$
\begin{array}{ll}
\left|\frac{v(\delta+p)(A-B)}{B}-1\right| \leq 1 \text { or }\left|\frac{v(\delta+p)(A-B)}{B}+1\right| \leq 1 & \text { if } B \neq 0 \\
|v(\delta+p) A| \leq \pi & \text { if } B=0 .
\end{array}
$$

If $f \in \mathcal{A}(p)$ with $H_{p, \eta, \mu}^{\lambda, \delta} f(z) \neq 0$ for all $z \in \mathbb{U}^{*}=\mathbb{U} \backslash\{0\}$, then:

$$
\frac{H_{p, \eta, \mu}^{\lambda, \delta+1} f(z)}{H_{p, \eta, \mu}^{\lambda, \delta} f(z)} \prec \frac{1+A z}{1+B z}
$$

implies:

$$
\left(\frac{H_{p, \eta, \mu}^{\lambda, \delta} f(z)}{z^{p}}\right)^{v} \prec q(z)
$$

where:

$$
q(z)= \begin{cases}(1+B z)^{v(\delta+p)(A-B) / B} & \text { if } B \neq 0 \\ e^{v(\delta+p) A z} & \text { if } B=0\end{cases}
$$

is the best dominant.

Proof. Putting:

$$
\Delta(z)=\left(\frac{H_{p, \eta, \mu}^{\lambda, \delta} f(z)}{z^{p}}\right)^{v}(z \in \mathbb{U})
$$


Then, $\Delta$ is analytic in $\mathbb{U}, \Delta(0)=1$ and $\Delta(z) \neq 0$ for all $z \in \mathbb{U}$. Taking the logarithmic derivatives on both sides of (34) and using (3), we have:

$$
1+\frac{z \Delta^{\prime}(z)}{v(\delta+p) \Delta(z)}=\frac{H_{p, \eta, \mu}^{\lambda, \delta+1} f(z)}{H_{p, \eta, \nu}^{\lambda, \delta} f(z)} \prec \frac{1+A z}{1+B z} .
$$

Now, the assertions of Theorem 5 follow by Lemma 4 .

Theorem 6. Let $0 \leq \alpha \leq 1, \zeta>1$. If $f(z) \in \mathcal{A}(p)$ satisfies:

$$
\Re\left((1-\alpha) \frac{\left(H_{p, \eta, \mu}^{\lambda, \delta+2} f(z)\right)^{\prime}}{\left(H_{p, \eta, \mu}^{\lambda, \delta+1} f(z)\right)^{\prime}}+\alpha \frac{\left(H_{p, \eta, \mu}^{\lambda, \delta+1} f(z)\right)^{\prime}}{\left(H_{p, \eta, \mu}^{\lambda, \delta} f(z)\right)^{\prime}}\right)<\zeta,
$$

then:

$$
\Re\left(\frac{H_{p, \eta, \mu}^{\lambda, \delta+1} f(z)}{H_{p, \eta, \mu}^{\lambda, \delta} f(z)}\right)<\beta,
$$

where $\beta \in(1, \infty)$ is the positive root of the equation:

$$
2(\delta+p+\alpha) \beta^{2}-[2 \zeta(\delta+p+1)-(1-\alpha)] \beta-(1-\alpha)=0 .
$$

Proof. Let:

$$
\frac{H_{p, \eta, \mu}^{\lambda, \delta+1} f(z)}{H_{p, \eta, \mu}^{\lambda, \delta} f(z)}=\beta+(1-\beta) \varphi(z) .
$$

Then, $\varphi$ is analytic in $\mathbb{U}, \varphi(0)=1$ and $\varphi(z) \neq 0$ for all $z \in \mathbb{U}$. Taking the logarithmic derivatives on both sides of (37) and using the identity (3), we have:

$$
(\delta+p+1) \frac{\left(H_{p, \eta, \mu}^{\lambda, \delta+2} f(z)\right)^{\prime}}{\left(H_{p, \eta, \mu}^{\lambda, \delta+1} f(z)\right)^{\prime}}-(\delta+p) \frac{\left(H_{p, \eta, \mu}^{\lambda, \delta+1} f(z)\right)^{\prime}}{\left(H_{p, \eta, \mu}^{\lambda, \delta} f(z)\right)^{\prime}}=1+\frac{(1-\beta) z \varphi^{\prime}(z)}{\beta+(1-\beta) \varphi(z)}
$$

and so:

$$
\begin{gathered}
(1-\alpha) \frac{\left(H_{p, \eta, \mu}^{\lambda, \delta+2} f(z)\right)^{\prime}}{\left(H_{p, \eta, \mu}^{\lambda, \delta+1} f(z)\right)^{\prime}}+\alpha \frac{\left(H_{p, \eta, \mu}^{\lambda, \delta+1} f(z)\right)^{\prime}}{\left(H_{p, \eta, \mu}^{\lambda, \delta} f(z)\right)^{\prime}}=\alpha \beta+\frac{(1-\alpha)(\delta+p) \beta}{\delta+p+1} \\
+\frac{(1-\beta)[\alpha+(1-\alpha)(\delta+p)]}{\delta+p+1} \varphi(z)+\frac{(1-\alpha)(1-\beta)}{[\beta+(1-\beta) \varphi(z)](\delta+p+1)} z \varphi^{\prime}(z) .
\end{gathered}
$$

Let:

$$
\begin{aligned}
\Psi(r, s ; z)= & \alpha \beta+\frac{(1-\alpha)(\delta+p) \beta}{\delta+p+1}+\frac{(1-\beta)[\alpha+(1-\alpha)(\delta+p)]}{\delta+p+1} r \\
& +\frac{(1-\alpha)(1-\beta)}{[\beta+(1-\beta) \varphi(z)](\delta+p+1)} s,
\end{aligned}
$$

and:

$$
\Omega=\{w \in \mathbb{C}: \Re(w)<\zeta\} .
$$


Then, for $x, y \leq-\frac{1+x^{2}}{2}$, we have:

$$
\begin{aligned}
\Re\{\Psi(i x, y ; z)\} & =\alpha \beta+\frac{(1-\alpha)(\delta+p) \beta}{\delta+p+1}+\frac{(1-\alpha)(1-\beta) \beta y}{\left[\beta^{2}+(1-\beta)^{2} x^{2}\right](\delta+p+1)} \\
& \geq \alpha \beta+\frac{(1-\alpha)(\delta+p) \beta}{\delta+p+1}-\frac{(1-\alpha)(1-\beta)}{2 \beta(\delta+p+1)}=\zeta,
\end{aligned}
$$

where $\beta$ is the positive root of Equation (36). Suppose that:

$$
R(\beta)=2(\delta+p+\alpha) \beta^{2}-[2 \zeta(\delta+p+1)-(1-\alpha)] \beta-(1-\alpha)=0 .
$$

For $\beta=0, R(0)=-(1-\alpha) \leq 0$ and for $\beta=1, R(1)=2(\delta+p)(1-\zeta)+2(\alpha-\zeta) \leq 0$. This proves that $\beta \in(1, \infty)$. Thus, for $z \in \mathbb{U}, \Psi(i x, y ; z) \notin \Omega$, and so, we obtain the required result by an application of Lemma 5 .

Theorem 7. Suppose that $0<\varepsilon_{1}, \varepsilon_{2} \leq 1$. If:

$$
-\frac{\pi}{2} \varepsilon_{1}<\arg \left\{(1-\theta) \frac{\left(H_{p, \eta, \mu}^{\lambda, \delta} f(z)\right)^{\prime}}{p z^{p-1}}+\theta \frac{\left(H_{p, \eta, \mu}^{\lambda, \delta+1} f(z)\right)^{\prime}}{p z^{p-1}}\right\}<\frac{\pi}{2} \varepsilon_{2},
$$

then:

$$
-\frac{\pi}{2} \xi_{1}<\arg \left(\frac{\left(H_{p, \eta, \mu}^{\lambda, \delta} f(z)\right)^{\prime}}{p z^{p-1}}\right)<\frac{\pi}{2} \xi_{2}
$$

where:

$$
\varepsilon_{1}=\xi_{1}+\frac{2}{\pi} \arctan \left(\frac{\left(\xi_{1}+\xi_{2}\right) \theta}{2(\delta+p)} \frac{1-|a|}{1+|a|}\right), \varepsilon_{2}=\xi_{2}+\frac{2}{\pi} \arctan \left(\frac{\left(\xi_{1}+\xi_{2}\right) \theta}{2(\delta+p)} \frac{1-|a|}{1+|a|}\right) .
$$

Proof. Let:

$$
\phi(z)=\frac{\left(H_{p, \eta, \mu}^{\lambda, \delta} f(z)\right)^{\prime}}{p z^{p-1}}(z \in \mathbb{U}) .
$$

Then, from Theorem 1, we have:

$$
(1-\theta) \frac{\left(H_{p, \eta, \mu}^{\lambda, \delta} f(z)\right)^{\prime}}{p z^{p-1}}+\theta \frac{\left(H_{p, \eta, \mu}^{\lambda, \delta+1} f(z)\right)^{\prime}}{p z^{p-1}}=\phi(z)+\frac{\theta z \phi^{\prime}(z)}{\delta+p} .
$$

Let $U(z)$ be the function that maps $\mathbb{U}$ onto the domain:

$$
\left\{w \in \mathbb{C}:-\frac{\pi}{2} \varepsilon_{1}<\arg (w)<\frac{\pi}{2} \varepsilon_{2}\right\},
$$

with $U(0)=1$, then:

$$
\phi(z)+\frac{\theta z \phi^{\prime}(z)}{\delta+p} \prec U(z) .
$$


Assume that $z_{1}, z_{2}$ are two points in $\mathbb{U}$ such that the condition (9) is satisfied, then by Lemma 6 , we obtain (10) under the constraint (11). Therefore,

$$
\begin{aligned}
\arg \left[(\delta+p) \phi\left(z_{1}\right)+\theta z_{1} \phi^{\prime}\left(z_{1}\right)\right] & =\arg \phi\left(z_{1}\right)\left[(\delta+p)+\theta \frac{z_{1} \phi^{\prime}\left(z_{1}\right)}{\phi\left(z_{1}\right)}\right] \\
& =\arg \phi\left(z_{1}\right)+\arg \left[(\delta+p)+\theta \frac{z_{1} \phi^{\prime}\left(z_{1}\right)}{\phi\left(z_{1}\right)}\right] \\
& =-\frac{\pi}{2} \xi_{1}+\arg \left[(\delta+p)-i \theta \frac{\left(\xi_{1}+\xi_{2}\right) \kappa}{2}\right] \\
& =-\frac{\pi}{2} \xi_{1}-\arctan \left[\frac{\left(\xi_{1}+\xi_{2}\right) \theta \kappa}{2(\delta+p)}\right] \\
& \leq-\frac{\pi}{2} \xi_{1}-\arctan \left[\frac{\left(\xi_{1}+\xi_{2}\right) \theta}{2(\delta+p)} \frac{1-|a|}{1+|a|}\right],
\end{aligned}
$$

and:

$$
\arg \left[(\delta+p) \phi\left(z_{2}\right)+\theta z_{2} \phi^{\prime}\left(z_{2}\right)\right] \geq \frac{\pi}{2} \xi_{2}+\arctan \left[\frac{\left(\xi_{1}+\xi_{2}\right) \theta}{2(\delta+p)} \frac{1-|a|}{1+|a|}\right] .
$$

which contradicts the assumption (38). This evidently completes the proof of Theorem 7.

Acknowledgments: The authors would like to thank all referees for their valuable comments which led to the improvement of this paper.

Author Contributions: All the authors read and approved the final manuscript as a consequence of the authors meetings.

Conflicts of Interest: The authors declare no conflict of interest.

\section{References}

1. Bulboacă, T. Differential Subordinations and Superordinations, Recent Results; House of Scientific Book Publ.: Cluj-Napoca, Romania, 2005.

2. Miller, S.S.; Mocanu, P.T. Differential Subordination: Theory and Applications, Series on Monographs and Textbooks in Pure and Applied Mathematics; Marcel Dekker Inc.: New York, NY, USA, 2000; Volume 225.

3. Owa, S. On the distortion theorems I. Kyungpook Math. J. 1978, 18, 53-59.

4. Owa, S.; Srivastava, H.M. Univalent and starlike generalized hypergeometric functions. Can. J. Math. 1987, 39, 1057-1077. [CrossRef]

5. Srivastava, H.M.; Saigo, M.; Owa, S. A class of distortion theorems involving certain operators of fractional calculus. J. Math. Anal. Appl. 1988, 131, 412-420. [CrossRef]

6. Prajapat, J.K.; Raina, R.K.; Srivastava, H.M. Some inclusion properties for certain subclasses of strongly starlike and strongly convex functions involving a family of fractional integral operators. Integral Transform. Spec. Funct. 2007, 18, 639-651. [CrossRef]

7. Goyal, S.P.; Prajapat, J.K. A new class of analytic $p$-valent functions with negative coefficients and fractional calculus operators. Tamsui Oxf. J. Math. Sci. 2004, 20, 175-186.

8. Prajapat, J.K.; Aouf, M.K. Majorization problem for certain class of $p$-valently analytic function defined by generalized fractional differintegral operator. Comput. Math. Appl. 2012, 63, 42-47. [CrossRef]

9. Tang, H.; Deng, G.; Li, S.; Aouf, M.K. Inclusion results for certain subclasses of spiral-like multivalent functions involving a generalized fractional differintegral operator. Integral Transform. Spec. Funct. 2013, 24, 873-883. [CrossRef]

10. Aouf, M.K.; Mostafa, A.O.; Zayed, H.M. Some characterizations of integral operators associated with certain classes of $p$-valent functions defined by the Srivastava-Saigo-Owa fractional differintegral operator. Complex Anal. Oper. Theory 2016, 10, 1267-1275. [CrossRef]

11. Aouf, M.K.; Mostafa, A.O.; Zayed, H.M. Subordination and superordination properties of $p$-valent functions defined by a generalized fractional differintegral operator. Quaest. Math. 2016, 39, 545-560. [CrossRef] 
12. Aouf, M.K.; Mostafa, A.O.; Zayed, H.M. On certain subclasses of multivalent functions defined by a generalized fractional differintegral operator. Afr. Mat. 2017, 28, 99-107. [CrossRef]

13. Mostafa, A.O.; Aouf, M.K.; Zayed, H.M.; Bulboacă, T. Multivalent functions associated with Srivastava-Saigo -Owa fractional differintegral operator. RACSAM 2017. [CrossRef]

14. Mostafa, A.O.; Aouf, M.K.; Zayed, H.M. Inclusion relations for subclasses of multivalent functions defined by Srivastava-Saigo-Owa fractional differintegral operator. Afr. Mat. 2018, -018-0567-3. [CrossRef]

15. Mostafa, A.O.; Aouf, M.K.; Zayed, H.M. Subordinating results for $p$-valent functions associated with the Srivastava-Saigo-Owa fractional differintegral operator. Afr. Mat. 2018. [CrossRef]

16. Mostafa, A.O.; Aouf, M.K. Some applications of differential subordination of $\mathrm{p}$-valent functions associated with Cho-Kwon-Srivastava operator. Acta Math. Sin. (Engl. Ser.) 2009, 25, 1483-1496. [CrossRef]

17. Wang, Z.; Shi, L. Some properties of certain extended fractional differintegral operator. RACSAM 2017, 1-11. [CrossRef]

18. Hallenbeck, D.Z.; Ruscheweyh, S. Subordination by convex functions. Proc. Am. Math. Soc. 1975, 52, 191-195. [CrossRef]

19. Pommerenke, C. Univalent Functions; Vandenhoeck \& Ruprecht: Göttingen, Germany, 1975.

20. Stankiewicz, J.; Stankiewicz, Z. Some applications of Hadamard convolution in the theory of functions. Ann. Univ. Mariae Curie-Sklodowska Sect. A 1986, 40, 251-265.

21. Obradović, M.; Owa, S. On certain properties for some classes of starlike functions. J. Math. Anal. Appl. 1990, 145, 357-364. [CrossRef]

22. Takahashi, N.; Nunokawa, M. A certain connection between starlike and convex functions. Appl. Math. Lett. 2003, 16, 653-655. [CrossRef]

23. Choi, J.H.; Saigo, M.; Srivastava, H.M. Some inclusion properties of a certain family of integral operators. J. Math. Anal. Appl. 2002, 276, 432-445. [CrossRef]

(C) 2018 by the authors. Licensee MDPI, Basel, Switzerland. This article is an open access article distributed under the terms and conditions of the Creative Commons Attribution (CC BY) license (http://creativecommons.org/licenses/by/4.0/). 